\title{
Unilateral Impact of Altered Loading by Changing Teeth Height on the TMJ Fibrocartilage: Disc and Condyle of Wistar Rats
}

\author{
Roxanne M. Olvera-Farias' ${ }^{1}$ Jose Raymundo Cruz-Perez², Rogelio Salinas-Gutierrez ${ }^{3}$, \\ Jose Antonio Guerrero-Diaz de Leon ${ }^{3}$, Juan B. Kouri-Flores², Raul Rosales-Ibañez ${ }^{4}$, \\ David Masuoka Ito ${ }^{5}$, Alma Lilian Guerrero Barrera ${ }^{6 *}$ \\ ${ }^{1}$ Departamento de Morfología, Universidad Autónoma de Aguascalientes, Aguascalientes, México \\ ${ }^{2}$ Departamento de Infectómica y Patogénesis Molecular CINVESTAV-IPN, Mexico City, Mexico \\ ${ }^{3}$ Departamento de Estadística, Universidad Autónoma de Aguascalientes, Aguascalientes, México \\ ${ }^{4}$ Departamento de Estomatología, Universidad Autónoma de San Luis Potosí, San Luis Potosi, México \\ ${ }^{5}$ Departamento de Estomatología, Universidad Autónoma de Aguascalientes, Aguascalientes, México \\ ${ }^{6}$ Laboratorio de Biología Celular y Tisular, Departamento de Morfología, Universidad Autónoma de \\ Aguascalientes, Aguascalientes, México \\ Email: *alguerre@correo.uaa.mx
}

Received 16 December 2015; accepted 11 April 2016; published 14 April 2016

Copyright (C) 2016 by authors and Scientific Research Publishing Inc.

This work is licensed under the Creative Commons Attribution International License (CC BY).

http://creativecommons.org/licenses/by/4.0/

c) (i) Open Access

\section{Abstract}

Temporomandibular joint (TMJ) is sensitive to loading and mechanical stress that provokes morphological changes produced by the impact in the occlusal plane. Here, this impact is evaluated in TMJ articular disc and articular cartilage using an in vivo model of unilateral occlusal plane impact and by analysis of serial tissue sections stained with Hematoxylin-Eosin (H-E) or with Masson trichrome technique. Thus, six groups of 5 Wistar rats $(200-250$ g) are subjected to biomechanical dental stimulation by placing unilateral resin occlusal interference, or unilateral tooth wear made by upper left molars artificial mechanical devastation (1 control and 2 experimental groups for each treatment). Each treatment is evaluated two times at 1 and 15 days post-treatment. By H-E staining, control groups show chondrocytes arrangement as several cord cell groups in comparison with the experimental groups, which show an arrangement in one cord cell along of articular disc. However, this yields no significant difference $(p<0.05)$ in cell number between control and experimental groups. In contrast, in articular cartilage chondrocytes are random distributed along the superficial zone in control groups, while in experimental groups cell-free regions are

\footnotetext{
${ }^{*}$ Corresponding author.
}

How to cite this paper: Olvera-Farias, R.M., et al. (2016) Unilateral Impact of Altered Loading by Changing Teeth Height on the TMJ Fibrocartilage: Disc and Condyle of Wistar Rats. Microscopy Research, 4, 20-31. 
observed in superficial zone. An image Blue hue analysis for trichrome stain is performed to quantify collagen; this shows a significant collagen decrease $(p<0.05)$ in almost all experimental groups compared with the controls. A degenerative process biomechanically induced by unilateral occlusal plane modification, causes cell and tissue changes on the TMJ structures that remain the degenerative changes observed in early osteoarthritis.

\section{Keywords}

\section{Articular Disc, Articular Cartilage, Dental Occlusion, Collagen, Temporomandibular Joint}

\section{Introduction}

Temporomandibular disorders (TMDs) are commonly orofacial diseases produced by temporomandibular joint (TMJ) alteration. TMD worldwide prevalence varies between $7 \%$ and $84 \%$ of the population within an age range of 3 - 74 years [1] and affects about 33\% of patients in USA [2] [3] showing many similarities to musculoskeletal disorders of other parts of the body [4]. TMD prevalence varies from $2 \%$ in Turkish patients to $68 \%$ in young polish adults [5]. In Mexico, TMD prevalence is high (46.1\% to $49.25 \%)$ affecting mainly young people (26.1\%) and shows a 3:1 proportion in females. Woman has a higher risk of TMJ muscular tenderness or pain than males because of hormonal, behavioral or psychological differences [5] [6].

TMJ is deteriorated with age, internal changes and trauma, causing pain and TMD. Its etiology includes alteration of the occlusal plane and generation of abnormal loads as prosthodontic and orthodontic treatments, bruxism and malocclusions [7] [8]. TMDs are group of intra-articular, periarticular and systemic heterogenic conditions, characterized by masticatory system dysfunction and pain [3].

TMJ is formed by the mandibular condyle, the mandibular fossa in the temporal bone and the articular disc [7] [8]. The compressive, tensional and mechanical force resistance of the disc is correlated with the extracellular matrix (ECM) structural organization and its biochemical composition made mainly by type I collagen fibers, even in the mature and hypertrophic zones wherein is accompanied by type $\mathrm{X}$ collagen. The condylar cartilage has anisotropy under tension consistent with the primary anteroposterior motion of the condyle, suggesting that the cartilage fibrous zone is comparable with the TMJ disc fibrocartilage. Both the fibrous zone of the condylar cartilage and TMJ disc has circumferentially and anteroposteriorly aligned collagen fibers, producing an anisotropic tissue under tension and shear. The articular collagen is arranged in a tridimensional stable and resistant network to both tension and shear forces [9]. The chondrocyte response to mechanical compression involves ECM interactions. The mechanical load affects the cell metabolism, proliferation and differentiation, because it plays a key role in cell growth, adaptation, architecture and tissular regeneration [10].

As is explained before, TMJ mechanical stress is a risk factor to develop degenerative pathologies, alterations in the occlusal plane generates abnormal loads. Because to this, here using an in vivo model of modified occlusal plane by unilateral occlusal interference or unilateral tooth wear, the microscopic changes in the chondrocyte and collagen distribution in TMJ and in the articular disc are studied.

\section{Methods}

\subsection{Experimental Animals}

The UASLP Ethics Committee approved all the experimental procedures. None of the specimens treated died of illness during study period. In the histological analysis made here not observe inflammation signs in any of the groups in the TMJ. Methodology is summarized in Figure 1. Briefly, thirty adult male Wistar rats of $200-250 \mathrm{~g}$ were used for the experimental work, they were maintained at $21^{\circ} \mathrm{C}-23^{\circ} \mathrm{C}$ during the experiment with a photoperiod of $12 \mathrm{~h}$. Feed and water were provided ad libitum. The Wistar rats were provided by the animal center of the Universidad Autónoma de Aguascalientes, México.

Six groups of 5 animals were made. Two treatments were done: resin occlusal interference and unilateral tooth wear. For both treatments a control group of 5 animals were made respectively. Two times of evaluation were made at one and 15 days after each treatment. One day timepoint was chosen because early symptoms are presented after an occlusal interference and early histological changes are suspected to appear. 


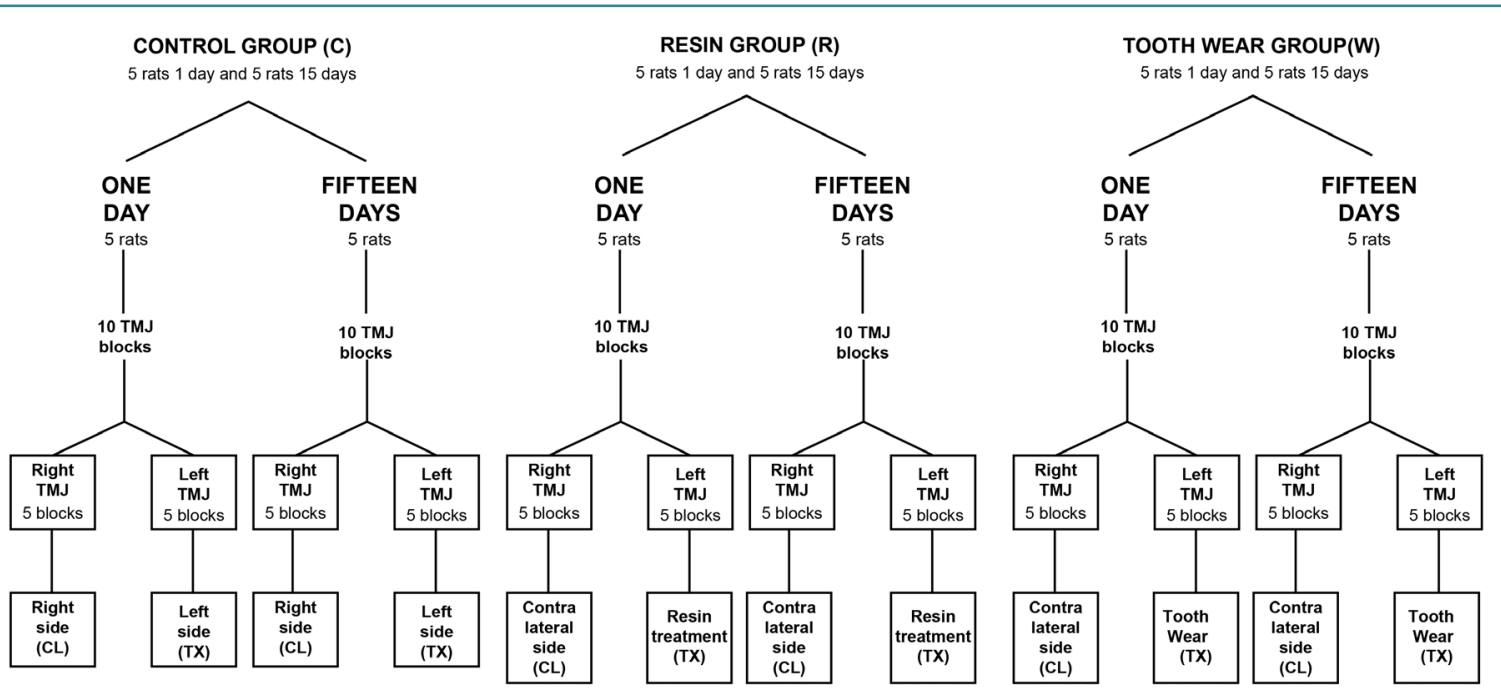

Figure 1. Flow diagram of experimental methodology.

For the clinical procedures the rats were anaesthetized with intraperitoneal injection of sodium pentobarbital (35 mg/kg body weight). The animals were euthanized at the end of each evaluation time using an overdose of intraperitoneal injection of sodium pentobarbital ( $60 \mathrm{mg} / \mathrm{kg}$ body weight).

After sacrifice, the obtained TMJ's were separated in left side (treatment side, TX) and right side (contralateral side, CL) as is described in tissue preparation. The animals did not show any behavioral changes, they continue eating at the same rate as the control and they did not display any signs on TMJ pain during the experimental time.

\subsection{Experimental Unilateral Occlusal Disorder}

As described before, two types of unilateral occlusal disorder were performed; resin occlusal interference and unilateral tooth wear (Figure 1) [11] [12]. The Resin unilateral occlusal interference (R group) was performed by making a small cavity with a high speed round bur \#1, then the enamel and dentin surfaces were etched with 37\% phosphoric acid (Prodensa R, México) for 20 seconds. The cavity was covered with the adhesive (TeEconom Ivoclar Vivadent, Asia). Finally, the cavity was filled with two layers of blue resin (Twinky Star, CE Voco, Germany); which was polymerized using a led curing light (Orisol, Miami, USA). One millimeter over the occlusal plane height verification was done with a graduated periodontal probe.

Unilateral tooth wear group (B group) was done by artificial mechanical devastation of superior left molars, using a high-speed diamond DR5 bur. The limit of the artificial mechanical devastation was until the cervical area (1 $\mathrm{mm}$ teeth bur down).

\subsection{Tissue Preparation}

Because to the shape of the articular disc, and in order to obtain sections extended properly, en bloc dissection was made. Also the condyles were analyzed because they are the most studied structure in OA and they were used to correlate the changes observed in this tissue with the articular disc.

Sixty TMJ blocks were obtained and histologically processed $(n=5)$. The decalcification and fixation of the tissue samples were done at the same time using a descaling and fixative solution (17\% EDTA/10\% Formalin). For decalcification the tissue samples were incubated with gently shaking during fifteen days in descaling and fixative solution.

The samples were divided in three groups: the control, unilateral tooth wear and unilateral resin occlusal interference groups. Dental treatments were done on the left side defining as treated side (TX) to this joint and the right side joint was defined as contralateral side (CL) (Figure 1).

After decalcification/fixation, samples were processed to embed in paraffin and then were cut with microtome (Leica RM 2125RT). The $5 \mu \mathrm{m}$ thick sections of TMJ in sagittal plane were cut and mounted in silanized-coated slides. For the analysis of articular disc and articular cartilage sections were divided in three parts, anterior, 
medial and posterior as describe as Zhang et al. [13]. The central section was selected for the analysis of each TMJ because this zone is the most affected in a biomechanical model.

\subsection{H-E Staining}

Histological methods were done according with the Armed Forces Institute of Pathology laboratory methods [14]. Deparaffinized tissues were submerged in hematoxylin during 2 minutes, after the slides were washed in distillated water for 1 minute. Immediately the slides were stained with Eosin for 2 minutes, and rinsed in distillated water during 5 minutes. Finally, the dehydration process was performed using three changes of one minute $96 \%$ alcohol followed by two changes of one minute with $100 \%$ alcohol and three changes of Xilol for one minute. The slides were mounted with Entellan (Merck Millipore, Darmstadt, Germany) to microscope observation (Leica, ICC50, Wetzlar, Germany).

\subsection{Masson Trichrome Staining}

Deparaffinized tissues were post fixated in Bouin solution during one hour at $56^{\circ} \mathrm{C}$. Then each slide was submerged in Ferric Hematoxylin solution during 15 minutes. After that, slides were stained with Biebrich Scarlet-Acid Fuchsin solution for 15 minutes. Then slides were incubated for fifteen minutes with Phosphotungstic Acid. Finally a Blue Anilin Staining procedure during 5 minutes was performed until bluish collagen fibers were observed. Between each stain washes with distillated water were performed. The dehydration was performed using three changes of one minute $96 \%$ alcohol followed by two changes of one minute with $100 \%$ alcohol and three changes of Xilol for one minute. The slides were mounted with Entellan (Merck Millipore, Darmstadt, Germany) to microscope observation (Leica, ICC50, Wetzlar, Germany).

\subsection{Image Analysis}

All the observations were made using a Leica DM1000 microscope (Wetzlar, Germany). Images were acquired with the Leica EC System (Wetzlar, Germany) at 20X (Trichromic Stain) and 100X (H-E) augmentation using LAS EZ (3.0 Software). Using Photoshop CS6 Adobe System LTD, USA/Software) articular disc and cartilage were divided sagittally in three equal parts: anterior, medial and posterior. For the analysis, only was evaluated the medial third of each section because to the form of the articular disc and also because that is the zone where the load is higher, as reported by Zhang [13]. The quantification of cells was made at the articular disc and for the superficial zone of articular cartilage, which are compound by fibrocartilage. The superficial zone was defined by the border between superficial and proliferative zones based on cell morphology and matrix composition. Nine images of each zone were evaluated. The quantification was made using $200 \times 200 \mu \mathrm{m}$ of the central area. To evaluate the thick of the articular disc and the superficial zone of articular cartilage nine images of each group were taken and three measures were done at central area, the average value obtained was used for statistical analysis.

All data was compared by one-way ANOVA. In all cases, p values less than 0.05 were considered statistically significant.

\subsection{Image and Statistical Analysis for Collagen Quantification}

Image analysis for trichrome stain was performed in order to quantify collagen and is summarized in Figure 2. The Blue hue represents the collagen in the tissue. The collagen quantification was performed in 9 samples stained with Masson's Trichrome Stain using the Blue hue analysis of the images obtained by microscopy. The measurement of blue hue collagen was focus on the articular disc and the superficial zone of the condylar cartilage. The image analysis was performed using RGB (red, green and blue color) and HSV (Hue, saturation and value) color spaces. Each image obtained was divided by one template of 100 squares of $12.5 \mu \mathrm{m} \times 12.5 \mu \mathrm{m}$. A randomized sample of $20 \%$ approximately was taken from each image and statistically analyzed using R Software [15]. For the image analysis in R, JPEG and biOps libraries were used. The different blue hues were separated using the HSV color space in a tone range of $100-200$ in the $\mathrm{H}$ channel. This range allowed to detect bluish tonalities and to eliminate the rest of the colors presents in the image. Once that the blue tones were filtrated, the HSV color space was transformed to RGB space with the finality of evaluate only the blue canal. Values were three times obtained in the RGB space ( 0 - 255 scale) for each treatment group. This method of 


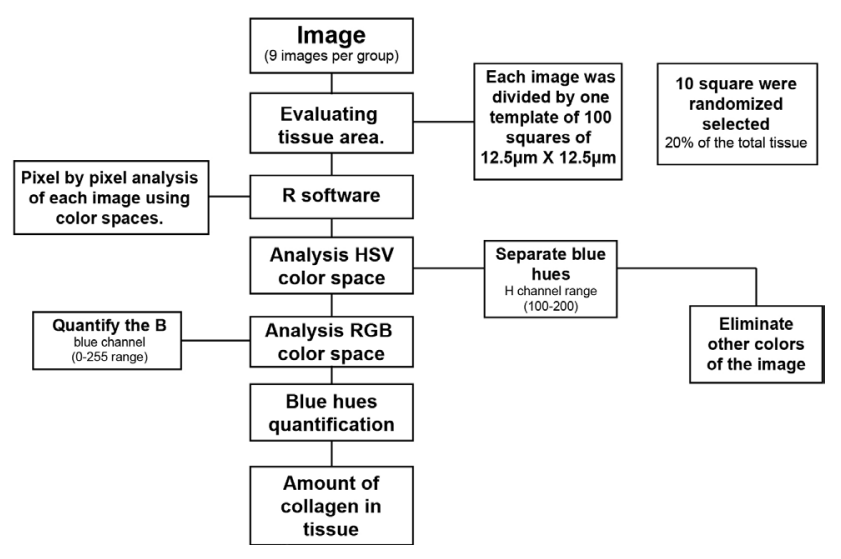

Figure 2. Flow diagram of collagen quantification method.

blue tone image analysis allows us to obtain a great amount of data because is working at pixel level.

Statistical analysis was performed using $\mathrm{R}$ Software [15]. Collagen quantification was expressed in each group $(n=9)$ blue color hue in the RGB space in average $+/$ - standard deviation, considering every special image selection pixels. Disc chondrocyte cell density and articular disc thickness were also average and standard deviation expressed for each group. All data was compared by one-way ANOVA and Tukey multi-comparison test method. In all cases, p values less than 0.05 were considered statistically significant.

\section{Results}

\subsection{Articular Disc Cell Morphology}

The articular disc in control group showed a normal composition of regular connective tissue and fibrocartilage. Also the morphology of chondrocytes was normal, oriented at the same direction that collagen fibers. Sometimes isolated or in a small cell cords arrangement. Cells showed rounded and big nuclei. There was high quantity of collagen fibers in the articular disc. In the experimental groups the articular disc presented similar cell morphology to the control group without signs of inflammation. However, an important finding was observed in the cell distribution in one cord cell arrangement along of articular disc (Figure 3).

\subsection{Articular Cartilage Cell Morphology}

In control groups the articular cartilage showed the five normal layers: fibrous or superficial, proliferative, intermediate, hypertrophic and calcified zones. The superficial layer was similar to articular disc with chondrocytes oriented in parallel and with normal structure. Collagen fibers also were organized as parallel fibers in anteroposterior direction (Figure 4). In the experimental groups articular cartilage was observed without any morphological changes in the treated side but the chondrocyte morphology became flattened with big and flattened nuclei in some contralateral groups (1R-CL, 1W-CL, 15R-CL and 15W-CL). The chondrocyte and collagen arrangement became irregular and deteriorate after 15 days of treatment in treated and contralateral sides (1R-CL, 1W-CL, 15R-CL and 15W-CL) (Figure 4).

\subsection{Articular Disc and Articular Cartilage Cell Quantification}

In articular disc the 1R-TX group showed a statistically significant but transitory increase (50\%) in articular disc cell number comparing with the control (Figure 5(a)). A statistically significant decrease (34\% - 46\%) in cellularity was found in the experimental fifteen days groups (15R and $15 \mathrm{~W}$, both sides) when comparing with 1RTX group (Figure 5(a)).

At 1 day, articular cartilage in tooth wear group in both TMJ presented a significant cell number increase (50\% - 63\%) in the superficial zone compared with control (Figure 5(b)). The 15R-TX and 15W-CL groups showed a significant cell density decrease (33\% - 42\%) when compared with 1W-TX. Independently of the quantitative analysis of this zone, at the end on the fifteen days in experimental groups, cell free areas were founded, reflected that a chondrocyte rearrangement happened. Cell clusters, which leaved place for cell free zones were 

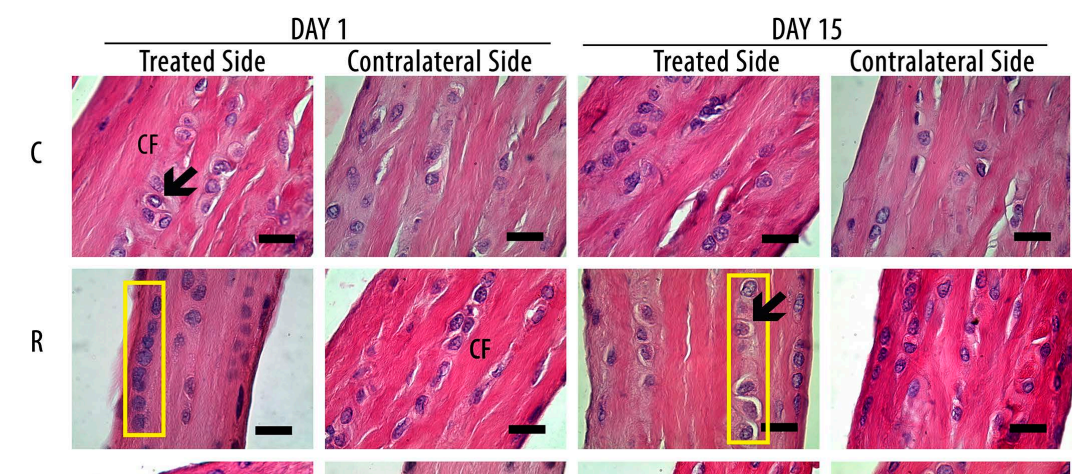

W
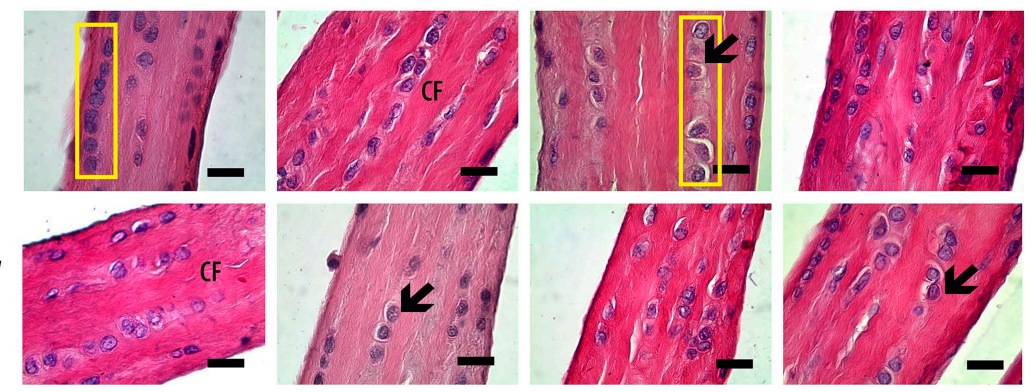

Figure 3. Histological Analysis of Articular Disc (X100). TMJ articular disc sagittal sections stained with H-E. The groups were designed with a letter: Control group ("C”), unilateral resin occlusal interference ("R") and unilateral tooth wear group ("W"). Scale Bar, $10 \mathrm{~m}$. The anterioposterior direction of the tissue is going from left to right. The cellular morphology in the experimental and control groups are similar. They showed a rounded nucleus (purple stained) with diminished cytoplasm (light pink around nuclei, marked with a black arrow). The collagen fibers (CF) are stained with intense pink, they constitute de major part of the articular disc tissue. The morphological analysis made with H\&E stain, showed in control groups, chondrocytes arrangement as several cord cell groups in comparison with the experimental groups which showed an arrangement in one cord cell along of articular disc (yellow rectangle).

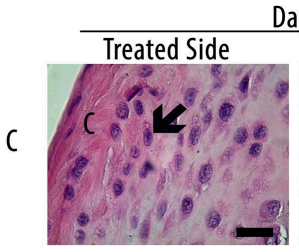

Day 1
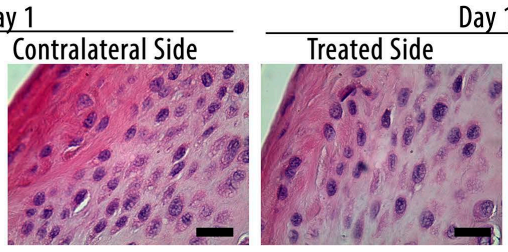

Day 15

$\mathrm{R}$
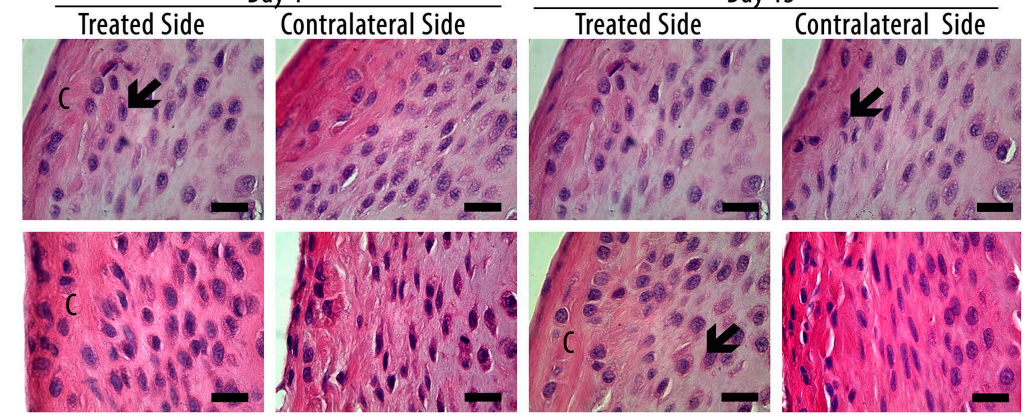

W
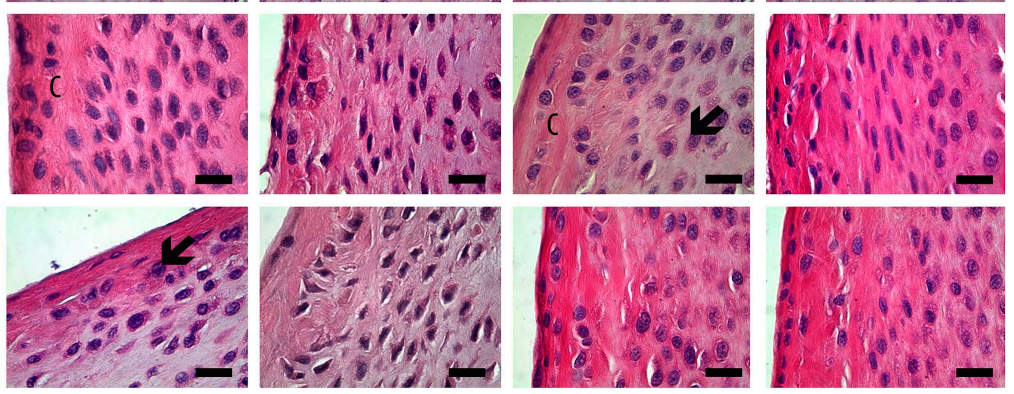

Figure 4. Histological Analysis of Articular Cartilage (X100). TMJ articular cartilage sagittal sections stained with H\&E. The groups were designed with a letter: Control group (“C”), unilateral resin occlusal interference ("R") and unilateral tooth wear group ("W"). Scale Bar 10 $\mu \mathrm{m}$. The anterioposterior direction of the tissue is going from left to right. The control group presented a rounded nucleus (black arrow), lightly tapered cellular morphology in a parallel distribution with collagenous fibers (CF). The experimental groups presented flattened cell morphology. In the day 15 of evaluation all the groups excepting the control, the cells changed their normal arrangement in the articular cartilage zones.

observed (Figure 5(b)).

\subsection{Articular Disc and Superficial Zone of Articular Cartilage Thickness Evaluation}

The 15W-TX group presented a significant decrease (32\%) in articular disc thickness in comparison to control 

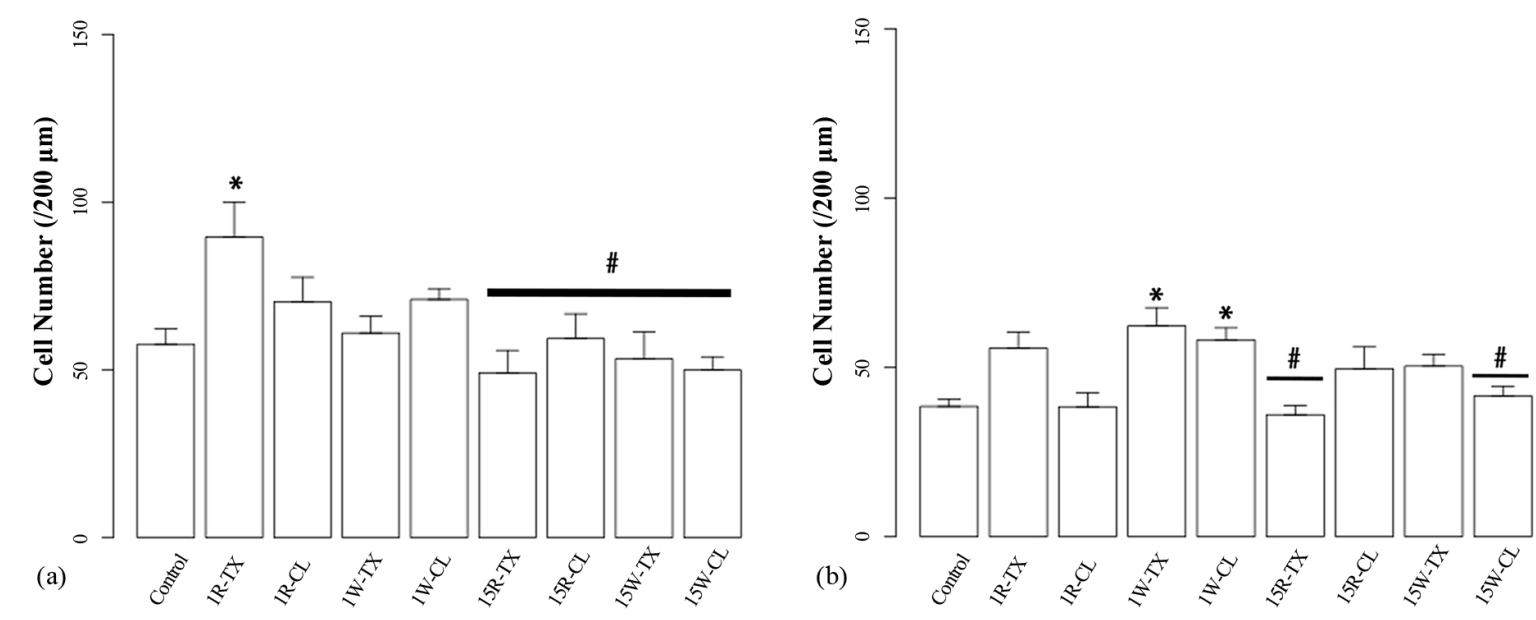

Figure 5. Articular disc and articular cartilage cell quantification. (a) Cell quantification analysis in articular disc. Control samples represents 1 and 15 days. The cell density in the $1 \mathrm{R}-\mathrm{TX}$ presented a significant increase ${ }^{*} \mathrm{p}<0.05$ compared with the control. The 15R-TX, 15R-CL, 15W-TX and 15W-CL had a significant decrease compared with the 1R-TX group. * p $<0.05$ when the values compared with 1R-TX. (b) Cell quantification analysis in articular cartilage superficial zone. The 1W-TX and $1 \mathrm{~W}-\mathrm{CL}$ group had a significant increase ${ }^{*} \mathrm{p}<0.05$ compared with control. The 15R-TX and 15W-CL groups presented a significant diminution compared with $1 \mathrm{~W}-\mathrm{T}$. ${ }^{\#} \mathrm{p}<0.05$, when the values compared with $1 \mathrm{~W}-\mathrm{TX}$.

group (Figure 6(a)). The unilateral tooth wear group (both TMJ) in the two evaluation time and the fifteen day unilateral resin occlusal interference (15R, both sides) showed a significant decrease (30\% - 44\%) in cell density when compared 1R-TX. Apparently articular disc thickness diminished with time after resin modified unilateral occlusal plane (Figure 6(a)).

An important thickness increase (31\% - 46\%) in the superficial zone of articular cartilage was found in the one and fifteen day tooth wear groups (1W and 15W, both sides) and 15R-CL respect the control (Figure 6(b)). The 15R-TX group showed thickness diminution (30\%) compared with one day of unilateral tooth wear group (1W, both sides) and the $15 \mathrm{~W}$-CL groups. There is general a similarity in the changes produced by mechanical stress due to the unilateral modification of the occlusal plane. However in the occlusal interference group the changes were observed in either treated or contralateral side (Figure 6(b)).

\subsection{Articular Disc Collagen Quantification}

Masson's Trichrome Stain shows with blue tones, the presence of collagen fibers (Figure 7). The staining showed a diminution in the blue intensity in the experimental groups (Figure 7(b)), also is observed that the articular disc diminished their thickness in the experimental groups. Some free cell zones were observed (Figure 7(b)), are marked by black arrows. The collagen quantification by RGB and HSV color spaces showed a decrease significantly $(2 \%-13 \%)$ in almost all the experimental groups, except in the 1R-TX there was no changes (Figure 7(a)). And in the 1R-CL the collagen quantity was significantly increased (2\%) when comparing to control group. However the collagen quantity significantly diminished (13\%) in the 1W-TX, 15W-CL, 15R-TX and 15R-CL groups compared with control (Figure 7(a)). By these findings is observed that the modified unilateral occlusal plane can provoke collagen amount changes even in a short times.

\section{Discussion}

Any no-compensated maxillomandibular alteration that causes an articular overload as occlusal interference and bruxism provokes TMJ alteration; both types of biomechanical stress in vivo model used here stimulated these two frequent conditions in dental practice [3]. The occlusal unilateral interference model was performed because usually the clinic occlusal interference is unilateral. Although the clinic bruxism usually is bilateral, in order to make comparable our experimental results, unilateral tooth wear (simulating a bruxism) model was applied in this study. The evaluation times were 1 or 15 days post-treatment, because we were interested in estimating the changes presented at short time. We hypothesized that the tooth wear group would have greater articular changes when compared to the occlusal interference group. Here was also expected that the articular damage was more 



Figure 6. Articular disc and superficial zone of articular cartilage thickness evaluation. (a) Thickness analysis of articular disc. Control samples represents 1 and 15 days. The 15W-TX group presented a significant decrease ${ }^{*} \mathrm{p}<0.05$ compared with control. The 1W, 15W and 15R both sides groups had a significant decrease compared with 1R-TX group. " p $<0.05$ when the values compared with 1R-CL. (b) Thickness analysis of articular cartilage superficial zone: The 1W-TX, 1W-CL, 15W-TX, 15W-CL and 15R-CL groups had a significant increase ${ }^{*} \mathrm{p}<0.05$ compared with control. ${ }^{\#} \mathrm{p}<0.05$ when the values compared with 15R-TX.

important in treated side comparing with contralateral side. However our findings show variable affection rate between treated and contralateral side at different time points and in-between groups. For that reason we rejected our hypothesis.

Wistar rats were choose as animal model, because their teeth not erupted continuously, and our results could be compared with other obtained in previous related studies that used the same model. In the model of modified occlusal plane in rabbit, expression changes produced by continuous masticatory functional loading were similar to osteoarthritis (OA). However, the rabbit teeth erupted continuously and provoked regression of the pre-altered occlusal plane over time [11]. In rat molars Sprague-Dawley, occlusal plane alteration, Zhang et al. observed degenerative changes in TMJ, as irregular array of chondrocytes, cell-free areas, increased synthesis of matrix metalloproteinases (MMPs), diminished chondrocyte proliferation, and subchondral bone loss [13]. Other dental occlusion modification studies did not show any articular cartilage modification when unilateral extractions or occlusal height were performed [12] [16].

The articular disc is similar to articular meniscus that plays a main role in the load distribution at articular compartments and in the mechanical shock absorption function. Meniscal tear, non-traumatic degeneration, meniscal damage or dysfunction causes OA [17]-[19]. Similarly, our results in vivo model of unilateral occlusal plane impact are showed in the treatment after 15 days degenerative changes as chondrocytes flattened morphology and collagen irregular arrangement. The degenerative changes observed with unilateral tooth wear are similar to changes found with unilateral extractions [12]. Mechanical stress in the TMJ produces damage in the articular disc and collagen decreasing related with the time in the experimental groups.

The most abundant component of ECM in the articular disc is collagen to estimate the total quantity of this protein detected by Masson's trichrome stain, a new easy technique is performed, using 2 color spaces. Thus, a collagen decrease is found in almost all the experimental groups, except in the 1R-TX, which shows no change. The collagen quantity in 1R-CL shows significant increased when comparing to control group, as is found in early TMJ inflammation of Sprague-Dawley rat in which also is increased aggrecan and glycosaminoglycan [20].

The collagen quantities decreased significantly in the unilateral tooth wear showed after 1 and 15 days, as well as happen in the resin occlusal interference group. In early knee OA provoked by mechanical stress similar changes are observed, where collagen type II concentration observed by quantitative PCR is decreased in superficial regions while in the deeper zones in reparation process showed an increasing in this kind of collagen [11] [21]. However, in our model more studies to determine the collagen types expressed are necessary. 
(a)
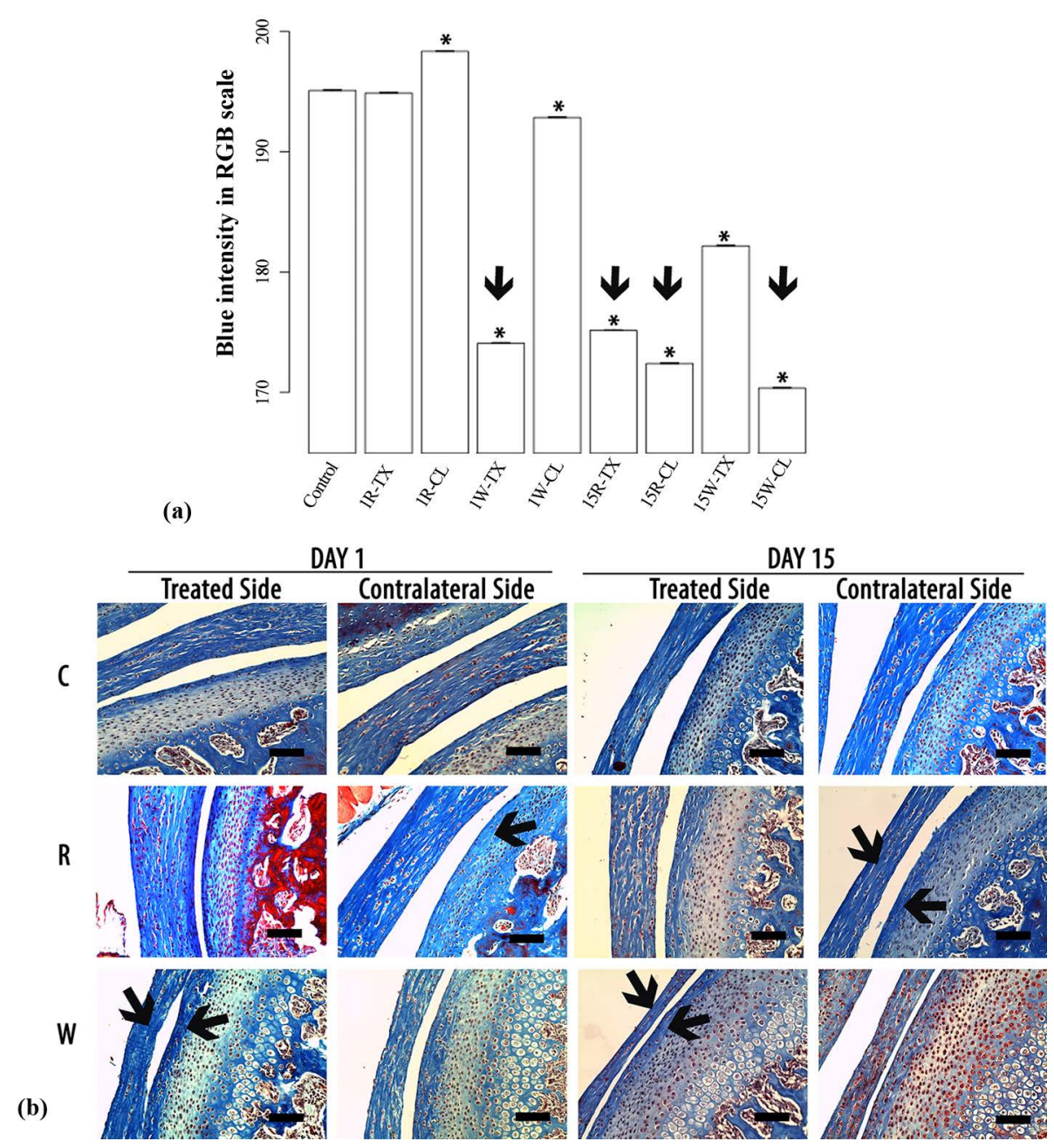

Figure 7. Articular disc collagen quantification (X20). (a) Collagen Quantification using Blue hue in RGB scale (160 - 200). This is a closer look of the original graph (0 - 255). Control samples represents 1 and 15 days. The 1R-CL group presented a significant increase ${ }^{*} \mathrm{p}<0.05$ compared with their control. The 1W-TX, 15R-TX, 15R-CL y 15W-CL groups showed noticeable diminution of the collagen compared with control group (shown with down arrow). (b) Histological Analysis of Articular Disc and Articular Cartilage. TMJ sagittal sections were stained with Masson's Trichrome Stain. The groups were designed with a letter: Control group (“C”), unilateral resin occlusal interference ("R") and unilateral tooth wear group ("W"). Scale Bar $50 \mu \mathrm{m}$. The staining showed a diminution in the blue intensity in the experimental groups. The articular disc diminished their thickness in the experimental groups. The articular cartilage presented a superficial zone thickened. We observed some free cells zones (indicated with a black arrow) in the articular disc and in articular cartilage superficial zone, in this last the cells were in clusters.

The synthesis of MMPs is also related with collagen decreasing, because they are the enzymes that degrades this protein [13] [20]. When the balance between ECM synthesis and its degradation fails, the water content is increased, the proteoglycan and type II collagen diminishes and finally the collagen network weakens [22]. Homeostasis then is important to avoid these changes [11], when the homeostasis of ECM production and chondrocyte proliferation fails $\mathrm{OA}$ is produced. The modified unilateral occlusal plane can provoke collagen amount changes even in short times, as our results showed. There are multiple regulatory pathways in chondrocytes that react to mechanical stimuli, including, signaling pathways and mechanisms that can directly lead to transcription, translation and postranslation changes, cell mediated extracellular organization and ECM degradation. The synovial joints can maintain intact load during normal life activities without develop OA. However, when the mechanical load exceeds the cartilage tolerance to repair and maintain itself; the cartilage damage plays an important role in development and progression of articular degeneration in different ways. An excessive surface contact stress can cause direct damage to articular cartilage and subchondral bone, and adversely alter the chondro- 
cyte function [22]. In our experiments, the number of chondrocytes in articular disc was the same as the control group, but clearly the metabolic stage of them was not enough to produce collagen, because was showed a less quantity of collagen, based in the blue hue of the Masson's Trichromic Stain, it could provoke decreased production of this protein.

The disorganization of collagen fibers in the superficial zone in articular cartilage produced by age is related to degenerative process. Our results showed articular cartilage surface parallel-organized collagen distribution in control groups whereas in condyle articular cartilage experimental groups, disorganization of collagen fibers was observed. In the knee meniscus, major changes have been observed with patient age increase when using Safranin O, showing a cell density diminution, mucoid degeneration and loss of collagen fiber organization [17], more studies at molecular level are necessary to show this kind of changes in our model.

The articular cartilage in the unilateral tooth wear group at 1 day of evaluation, a significantly increased number of chondrocytes at the superficial zone was observed, while the 15 days unilateral tooth wear group remain as the control. The $50 \%$ increase in cell number in the 1R-TX of the articular disc perhaps is due to changes to the tissue structure that would result in more cells in the central region. The cellular clusters are a characteristic of early reparation in the different layers of OA articular cartilage, where the chondrocyte mechanical stressed modified their ECM production [14]. Here, experimental groups showed cellular clusters as cord cells groups and cell-free areas in articular fibrocartilage and changes in the changes in the amount of matrix components. The mechanical stress on TMJ produced some scattered cell-free areas in articular cartilage, similar to degenerative changes or early OA-like lesions at 12 weeks after occlusal plane alteration [13] [23] [24]. The cellular clusters can adopt a different distribution from the typical OA accumulations. Pauli et al. induced a knee OA model and studied the knee menisci in which they noted a decrease in cell density, mucoid degeneration associated with some loss of collagen fiber organization and presence of cell aggregates. Although not resembling the typical OA cell clusters, which indicated a local fibro-chondrocyte proliferation or pseudo-cloning. The appearance of these clusters was as cellular alignments [17]. Our results suggest that mechanical stressed TMJ articular disc present the same phenomenon, showing cell distribution in one cord cell arrangement along of articular disc.

In the articular disc also was observed a marked diminution in the thickness in the 15W-TX compared with control group, instead the other groups that showed any differences. Unilateral tooth wear is an intense mechanical stress compared with occlusal interference because was produced an imbalance of the occlusal plane, and that is the reason for this change at 15 days post-treatment. When the articular cartilage thickness was analyzed in the resin occlusal interference group at 1 and 15 days any changes was observed, except for 15R-CL group, which their thickness increased significantly respect the control, this seems be related with the significant increased of collagen that this group also present, as Wang et al. observed, they related these changes with a hypertrophic response to mechanical stress [20]. Also unilateral tooth wear group in both evaluation times showed a significantly rise of thickness when was compared with control. Stimulated bruxism produces a high-speed modification in the thickness of the articular cartilage, showing the importance of relation of the occlusal plane with the TMJ. Yoshikawa et al. observed with experiment of occlusal plane alteration in articular cartilage at 3 day after treatment a thinning structure, they also observed cell cluster formation, as is showed in our results, they found that after 1 week this thinning disappear, then after 2 weeks of treatment they observed an increased thickness [11].

Is important for the dentist identify occlusal disorders in their patients in order to establish an immediately treatment ranging from self-care conservative treatment to surgical treatment when needed. In this study were observed degenerative changes in short term, so the treatment in time can prevent degenerative pathologies [13].

There are numerous studies that evaluate the superficial zone articular cartilage chondrocyte damage in the TMD which ones had proved to have a main role in the OA development [13] [25].

Modified unilateral occlusal plane at 1 and 15 days after treatment provoked a decreased collagen quantity and tissue thickness decreasing in articular disc. This was correlated with the articular cartilage degenerative changes in their TMJ. The mechanical stress induced an arrangement in cord cells in both occlusal interference or unilateral tooth wear. The unilateral tooth wear produced similar changes that found with unilateral extractions. The HSV and RGB color spaces analysis represent a new semi-quantitative method to quantify collagen when thricrome stain is used. Finally, the modified unilateral occlusal plane can induce morphological and structural changes in both articular cartilage and articular disc and they can act as an initial inductor of developing degenerative pathology. 


\section{Acknowledgements}

Roxanne Mirella Olvera Farias was supported by CONACYT scholarship No. 256312.

\section{References}

[1] Luther, F. (2007) TMD and Occlusion Part II. Damned If We Don't? Functional Occlusal Problems: TMD Epidemiology in a Wider Context. British Dental Journal, 202, 38-39. http://dx.doi.org/10.1038/bdj.2006.123

[2] De Laat, A. and Macaluso, G.M. (2002) Sleep Bruxism Is a Motor Disorder. Movement Disorders, 17, 67-69. http://dx.doi.org/10.1002/mds.10064

[3] Manfredini, D., Peretta, R., Guarda-Nardini, L. and Ferronato, G. (2010) Predictive Value of Combined Clinically Diagnosed Bruxism and Occlusal Features for TMJ Pain. Cranio: The Journal of Craniomandibular Practice, 28, 105113. http://www.maneyonline.com/doi/pdfplus/10.1179/crn.2010.015 http://dx.doi.org/10.1179/crn.2010.015

[4] Michalak, M., Paulo, M., Bożyk, A., Zadrożny, Ł., Wysokińska-Miszczuk, J., Michalak, I. and Borowicz, J. (2013) Incidence of Abnormalities in Temporomandibular Joints in a Population of 1100 Urban and Rural Patients Lacking Teeth and Other Parafunctions in 2003-2008. An International Problem. Annals of Agricultural and Environmental Medicine, 20, 86-90.

[5] Sánchez-Pérez, L., Irigoyen-Camacho, M.E., Molina-Frechero, N., Mendoza-Roaf, P., Medina-Salo, L.A., Hoyland, J. and Thomas, J.T. (1996) The Expression of Types X and VI Collagen and Fibrillin in Rat Mandibular Condylar Cartilage. Response to Mastication Forces. Acta Odontologica Scandinavica, 54, 295-302. http://dx.doi.org/10.3109/00016359609003541

[6] Casanova-Rosado, J.F., Medina-Solís, C.E., Vallejos-Sánchez, A.A., Casanova-Rosado, A.J., Hernández-Prado, B. and Avila-Burgos, L. (2006) Prevalence and Associated Factors for Temporomandibular Disorders in a Group of Mexican Adolescents and Youth Adults. Clinical Oral Investigations, 10, 42-49. http://dx.doi.org/10.1007/s00784-005-0021-4

[7] Celenza, F.V. and Nasedkin, J.N. (1978) Occlusion: The State of the Art. Quintessence Pub. Co., Chicago.

[8] Choi, K.Y., Yang, J.D., Chung, H.Y. and Cho, B.C. (2012) Current Concepts in the Mandibular Condyle Fracture Management Part I: Overview of Condylar Fracture. Archives of Plastic Surgery, 39, 291-300. http://dx.doi.org/10.5999/aps.2012.39.4.291

[9] Kuroda, S., Tanimoto, K., Izawa, T., Fujihara, S., Koolstra, J.H. and Tanaka, E. (2009) Biomechanical and Biochemical Characteristics of the Mandibular Condylar Cartilage. Osteoarthritis and Cartilage, 17, 1408-1415. http://dx.doi.org/10.1016/j.joca.2009.04.025

[10] Guilak, F., Alexopoulos, L.G., Upton, M.L., Youn, I., Choi, J.B., Cao, L. and Haider, M.A. (2006) The Pericellular Matrix as a Transducer of Biomechanical and Biochemical Signals in Articular Cartilage. Annals of the New York Academy of Sciences, 1068, 498-512. http://dx.doi.org/10.1196/annals.1346.011

[11] Yoshikawa, S., Nodai, E., Habu, M., Furuta, N., Fukuda, J. and Yamaguchi, K. (2008) Influence of Altered Occlusal Plane on Rabbit Temporomandibular Joint Cartilage. Journal of Oral Pathology and Medicine, 37, 30-37. http://dx.doi.org/10.1111/j.1600-0714.2007.00577.x

[12] Kikuta, T. (1980) A Histopathological Study of the Temporomandibular Joint Following Unilateral Loss of Occlusion in the Rhesus Monkey. Journal of the Kyusyu Dental Society, 34, 254-274.

[13] Zhang, M., Zhang, J., Lu, L., Qiu, Z.Y., Zhang, X., Yu, S.B., Wu, Y.P. and Wang, M.Q. (2013) Enhancement of Chondrocyte Autophagy Is an Early Response in the Degenerative Cartilage of the Temporomandibular Joint to Biomechanical Dental Stimulation. Apoptosis, 18, 423-434. http://dx.doi.org/10.1007/s10495-013-0811-0

[14] Prophet, E.B., Mills, B., Arrington, J.B. and Sobin, L.H. (1994) Armed Forces Institute of Pathology: Laboratory Methods in Histotechnology. Armed Forces Institute of Pathology, American Registry of Pathology, Washington DC. http://babel.hathitrust.org/cgi/pt?id=mdp.39015029715979;view=1up;seq=6

[15] Software R Core Team (2012) R: A Language and Environment for Statistical Computing. R Foundation for Statistical Computing, Vienna. http://www.R-project.org/

[16] Toyoshima, Y., Iizuka, T., Aita, H. and Ohata, N. (2003) Effect of Experimental Traumatic Occlusion on Temporomandibular Joint. Journal of Oral Biology, 45, 407-417. http://dx.doi.org/10.2330/joralbiosci1965.45.407

[17] Pauli, C., Grogan, S.P., Patil, S., Otsuki, S., Hasegawa, A., Koziol, J. and D’Lima, D.D. (2011) Macroscopic and Histopathologic Analysis of Human Knee Menisci in Aging and Osteoarthritis. Osteoarthritis and Cartilage, 9, 1132-1141. http://dx.doi.org/10.1016/j.joca.2011.05.008

[18] Englund, M., Guermazi, A. and Lohmander, L.S. (2009) The Meniscus in Knee Osteoarthritis. Rheumatic Disease Clinics of North America, 35, 579-590. http://dx.doi.org/10.1016/j.rdc.2009.08.004 
[19] Badlani, J.T., Borrero, C., Golla, S., Harner, C.D. and Irrgang, J.J. (2013) The Effects of Meniscus Injury on the Development of Knee Osteoarthritis: Data from the Osteoarthritis Initiative. American Journal of Sports Medicine, 41, 1238-1244. http://dx.doi.org/10.1177/0363546513490276

[20] Wang, G.W., Wang, M.Q., Wang, X.J., Yu, S.B., Liu, X.D. and Jiao, K. (2010) Changes in the Expression of MMP-3, MMP-9, TIMP-1 and Aggrecan in the Condylar Cartilage of Rats Induced by Experimentally Created Disordered Occlusion. Archives of Oral Biology, 55, 887-895. http://dx.doi.org/10.1016/j.archoralbio.2010.07.013

[21] Lahm, A., Mrosek, E., Spank, H., Erggelet, C., Kasch, R., Esser, J. and Merk, H. (2010) Changes in Content and Synthesis of Collagen Types and Proteoglycans in Osteoarthritis of the Knee Joint and Comparison of Quantitative Analysis with Photoshop-Based Image Analysis. Archives of Orthopaedic and Trauma Surgery, 130, 557-564. http://dx.doi.org/10.1007/s00402-009-0981-y

[22] Heijink, A., Gomoll, A.H., Madry, H., Drobnič, M., Filardo, G., Espregueira-Mendes, J. and Van Dijk, C.N. (2012) Biomechanical Considerations in the Pathogenesis of Osteoarthritis of the Knee. Knee Surgery, Sports Traumatology, Arthroscopy, 20, 423-435. http://dx.doi.org/10.1007/s00167-011-1818-0

[23] Sun, L., Wang, M., He, J., Liu, L., Chen, S. and Widmalm, S.E. (2009) Experimentally Created Nonbalanced Occlusion Effects on the Thickness of the Temporomandibular Joint Disc in Rats. Angle Orthodontist, 79, 51-53. http://dx.doi.org/10.2319/091907-450.1

[24] Wang, X.D., Kou, X.X., Mao, J.J., Gan, Y.H. and Zhou, Y.H. (2012) Sustained Inflammation Induces Degeneration of the Temporomandibular Joint. Journal of Dental Research, 91, 499-505. http://dx.doi.org/10.1177/0022034512441946

[25] Saarakkala, S., Julkunen, P., Kiviranta, P., Makitalo, J., Jurvelin, J.S. and Korhonen, R.K. (2010) Depth-Wise Progression of Osteoarthritis in Human Articular Cartilage: Investigation of Composition, Structure and Biomechanics. Osteoarthritis and Cartilage, 18, 73-81. http://dx.doi.org/10.1016/j.joca.2009.08.003 